\title{
Curricula 2001: A Brief Summary
}

\author{
Francis C.M. Lau \\ Vice President, Chapters Activities \\ IEEE Computer Society \\ The University of Hong Kong \\ f.lau@computer.org
}

\author{
Carl K. Chang \\ Vice President, Press Activities \\ IEEE Computer Society \\ The University of Illinois at Chicago \\ ckchang@eecs.uic.edu
}

\begin{abstract}
Model curricula are important resources for curricula planning and design. A joint task force between IEEE-CS and $A C M$ was formed to undertake the responsibility of revising the 1991 model curricula. The effort is now underway, and the result will be known as Curricula 2001.
\end{abstract}

\section{Introduction}

Since the pioneer effort by ACM in 1968, model computer curricula have played a key role in the design and implementation of computing programs in many institutions around the world. As computing is a rapidly changing field, any computing curricula cannot stay unchanged for too long. The latest such model, Computing Curricula 1991 (CC-91) by ACM and IEEE-CS, will be close to a decade old by the turn of the century, and it seems imperative that a replacement must soon be produced.

Although in a decade's time, technologies might have advanced by leaps and bounds, much of the basics of computing remain as valid and essential in any computing program as they ever have been. The new curricula, therefore, will be more a revision and enhancement than an overhaul.

A joint task force between IEEE-CS and ACM was formed in 1998 to take up the challenge of producing a revised model curricula by 2001. It is a challenge indeed because ever since the debut of the World Wide Web, information technology has become an inseparable part of everyone's life, and the application of computing has expanded in scope substantially, covering new grounds in business, education, news and media, entertainment, and so on. It is a challenge also because of the increasing need to carefully identify a reasonably small set of "core" materials that can be accommodated in all computing curricula, and which is not to be diluted because of the proliferation of new materials.

The official charter of the joint task force is
- to review CC-91 and develop a revised and enhanced version for the year 2001 (CC-2001) that will encompass the latest developments of computing technologies in the past decade and endure through the next decade.

A key reference for the effort is [1], and the official Web site for the effort is at

http: / /www. computer .org/educate/cc2001/

The task force began its work with a Web-based survey. 124 responses were received, of which $98 \%$ supported the idea of updating CC-91. The survey results indicated that knowledge units are useful, new areas should be added, a minimal core is necessary (if there is a good definition of what a core is), more models curricula should be developed to suit the varieties of different programs, and attention should be paid to accreditation criteria (in particular those by ABET and CSAB).

\section{Ten Principles}

In order to ensure lasting effects, the work of the task force is founded upon 10 important principles.

P1: Future computing curricula should have dual objectives: to produce both excellent researchers and excellent practitioners.

P2: Computing integrates mathematics, science, and engineering.

P3: Knowledge units are valuable in the process of curriculum design.

P4: Curricula 2001 must offer guidance in individual course design.

P5: Curricula 2001 must identify core concepts that should be presented to all students.

P6: Curricula 2001 must provide guidelines for courses beyond the required core. 
P7: Curricula 2001 must be international in scope.

P8: The development of Curricula 2001 must involve significant industry participation.

P9: Curricula 2001 must consider professional practice skills.

P10: Curricula 2001 must meet the need of undergraduate programs.

\section{Focus Groups}

The development of Curricula 2001 is an immense undertaking. A proper division of effort is crucial. At the top level, the task force's steering committee decided on a division between knowledge areas and pedagogy. Knowledge areas focus groups (KFGs) and pedagogy focus groups (PFGs) were formed as a result, and many volunteers having expertise in different subject areas relevant to these groups have since become members of these groups.

\subsection{KFGs}

The steering committee has identified 14 subject areas, each of which has been assigned a KFG. The 14 subject areas are:

\begin{tabular}{|rl|}
\hline 1. & Discrete Structures (DS) \\
2. & Programming Fundamentals (PF) \\
3. & Algorithms \& Complexity (AL) \\
4. & Languages and Translation (LT) \\
6. & Architecture (AR) \\
7. & Intelligent Systems (IS) \\
8. & Information Management (IM) \\
9. & Human-Computer Interaction (HC) \\
10. & Graphics and Visualization (GV) \\
11. & Net-centric Computing (NC) \\
12. & Software Engineering (SE) \\
13. & Computational Science (CN) \\
14. & Social \& Professional Issues (SP) \\
\hline
\end{tabular}

Each KFG is charged with the task to define the scope for the subject area for which the group is responsible, produce the associated list of knowledge units, separate the knowledge units into two or three levels (core-intermediateadvanced), and based on the knowledge units, suggest courses and corresponding lecture or lab hours.

\subsection{PFGs}

A number of pedagogical issues cut across the knowledge areas. This orthogonal dimension is missing in the
CC-91. The CC-2001 steering committee found these issues to be of such an importance today that their inclusion in Curricula 2001 is a must. Six PGFs have been formed to consider these issues and to eventually produce the corresponding guidelines and recommendations: The First Year (FY), Supporting Material (SM), The Computing Core (CC), Professional Practices (PP), Advanced Study (AS), and Computing across Curricula (AC).

\section{Curricula for the World}

One of the underlying principles for the task force to adhere to throughout is that Curricula 2001 must be applicable to the international scene. An international panel consisting of members from various representative countries has thus been formed. This panel will help keep Curricula 2001 in line with the principle by initiating a process that will bring in the necessary international influences to the design of the curricula.

Accreditation is another concern when international programs are in view. ABET (Accreditation Board for Engineering \& Technology) and CSAB (Computer Science Accreditation Board), although dominant in the US, are not necessarily the favored bodies in other countries. CC-2001 will need to be careful not putting all the emphasis on ABET and $\mathrm{CSAB}$.

\section{CS, CE, SE}

Curricula 2001, like CC-91, will probably be most relevant for "computer science" (CS) or CS-related programs. The other major computing programs include computer engineering (CE) and software engineering (SE). Because of the curricula effort (by the Software Engineering Coordinating Committee) that is going on independent of CC-2001 on software engineering education, CC-2001 will not have an enlarged component on SE education, but will maintain an interface to that other effort. CE is not as well developed as SE in terms of body of knowledge and as a profession. CC2001 will want to see more input from CE communities as to what Curricula 2001 should offer in terms of CE-specific recommendations. As far as the core for these different but closely related programs is concerned, the computing core of Curricula 2001 is targeted to be minimal enough that it can be included verbatim as core materials in any $\mathrm{CS}, \mathrm{CE}$, or SE program.

\section{References}

[1] C.K. Chang et al., "Curricula 2001: Bringing the Future to the Classroom", IEEE Computer, September $1999,85-89$. 